\title{
Predictors of Early Diabetic Retinopathy Regression with Ranibizumab in the RIDE and RISE Clinical Trials
}

This article was published in the following Dove Press journal: Clinical Ophthalmology

\author{
Michael Singer' \\ Mimi Liu ${ }^{2}$ \\ Patricio G Schlottmann ${ }^{3}$ \\ Arshad M Khanani ${ }^{4}$ \\ Miranda Hemphill ${ }^{5}$ \\ Lauren Hill $\mathbb{1}^{5}$ \\ Lisa Tuomi ${ }^{5}$ \\ Zdenka Haskova (D) ${ }^{5}$ \\ 'Medical Center Ophthalmology \\ Associates, San Antonio, TX, USA; \\ ${ }^{2}$ Colorado Retina Associates, Denver, \\ CO, USA; ${ }^{3}$ Organizacion Medica de \\ Investigacion, Buenos Aires, Argentina; \\ ${ }^{4}$ Sierra Eye Associates, Reno, NV, USA \\ ${ }^{5}$ Genentech, Inc., South San Francisco, \\ CA, USA
}

Correspondence: Michael Singer Medical Center Ophthalmology Associates, 9157 Huebner Road, San Antonio, TX 78240, USA

Tel +I 2102693754

Fax + I 210 5587679

Email msingerII@me.com
Purpose: To investigate the predictors of early diabetic retinopathy (DR) improvement in the RIDE/RISE (NCT00473382/NCT00473330) clinical trials.

Patients and Methods: In RIDE/RISE, adult patients with vision loss due to diabetic macular edema (DME) were randomized to monthly intravitreal ranibizumab 0.3 or $0.5 \mathrm{mg}$ $(n=502$ total) or sham $(n=257)$. DR severity was graded (using the Early Treatment Diabetic Retinopathy Study Diabetic Retinopathy Severity Scale). In this post hoc analysis of RIDE/ RISE, eyes with baseline DR score $\geq 35$ were evaluated for $\geq 2$-step improvements, and eyes with baseline DR score $\geq 43$ were evaluated for $\geq 3$-step improvements. The characteristics associated with $\geq 2$ - or $\geq 3$-step DR improvement at months 3 or 6 were assessed using univariate and/or multivariable analyses.

Results: The percentage of eyes with $\mathrm{a} \geq 2$ - or $\geq 3$-step DR improvement was $20.1 \%$ and $3.7 \%$ at month 3 and $31.2 \%$ and $5.8 \%$ at month 6 . Odds of $\geq 2$-step DR improvement at months 3 or 6 were significantly greater in eyes with moderately severe to severe nonproliferative DR (NPDR) at baseline versus less severe or more severe DR (both $P<0.0001$ ). At month 6 , odds of $\geq 2$-step DR improvement were significantly greater in eyes with no DME at month $3(P=0.008)$. Most patients with $\geq 3$-step DR improvement at months 3 or 6 had proliferative DR (PDR) at baseline $(83.3 \%$ and $66.7 \%)$.

Conclusion: The strongest predictors of DR response to ranibizumab at month 6 were baseline DR severity and DME quiescence at month 3. Eyes with the most robust early improvements had moderately severe or severe NPDR or PDR at baseline.

Keywords: diabetic retinopathy, ranibizumab, anti-VEGF, diabetic macular edema, post hoc

\section{Plain Language Summary}

Diabetic retinopathy (DR) is a vision-threatening complication of diabetes. It can range in severity from mild, moderate, or severe nonproliferative DR (NPDR) to proliferative DR (PDR; the most severe form of the disease). The most common cause of vision loss in DR is the development of fluid around the light-sensitive cells in the center of the retina (called diabetic macular edema $[\mathrm{DME}])$. Effective treatments are available, but not all patients achieve the same level or speed of improvement following treatment with the same drug. The purpose of this study was to take a look back at the clinical trials that first demonstrated the efficacy of ranibizumab in eyes with DR and DME in order to determine which factors made it more likely that a patient would achieve a rapid, robust improvement in DR severity during treatment with ranibizumab. This study found that patients with the most robust early improvements in DR severity (by month 6) were more likely to have had moderately severe or severe NPDR or PDR at the start of treatment (rather than less severe DR) and had their DME disappear after just 3 months of treatment. 


\section{Introduction}

Diabetic retinopathy (DR) is the leading cause of impaired vision among the working-age adult population in developed countries. ${ }^{1,2}$ It is an insidiously progressive disease that can worsen over time to proliferative DR (PDR) and can lead to vision-threatening complications such as diabetic macular edema (DME; the leading cause of vision loss in DR). ${ }^{2,3}$ The Diabetes Control and Complications Trial and the United Kingdom Prospective Diabetes Study showed that many patients diagnosed with DR continue to progress to vision loss despite diligent monitoring and control of blood glucose concentration. ${ }^{4-6}$

Vascular endothelial growth factor (VEGF) is one of the key factors mediating the underlying pathology of DR and DME. ${ }^{3,7-9}$ Several clinical trials have shown that intravitreal injections of anti-VEGF agents reduce DR progression and produce clinically and statistically significant improvements in DR severity. ${ }^{10-21}$ Among eyes with DR and DME treated with the anti-VEGF agent ranibizumab in the RIDE (NCT00473382) and RISE (NCT00473330) clinical trials, a significant percentage exhibited an at least 2- or 3-step improvement from baseline in DR severity as measured by the Early Treatment Diabetic Retinopathy Study (ETDRS)
Diabetic Retinopathy Severity Scale (DRSS). ${ }^{12,21-23}$ Approximately $20 \%$ and $4 \%$ achieved an at least 2 - or 3-step improvement, respectively, by month 3 , and the percentage of DR responders steadily increased with monthly treatment (Figure 1; reproduced with permission from Sun et al 2019). ${ }^{21}$ The proportion of eyes achieving a 2-step or greater DR improvement with ranibizumab was statistically significant compared with sham injections at all follow-up visits. ${ }^{20}$ This is clinically important because DR regression of 2 steps or more is accompanied by marked improvements in vision and is associated with a high rate of DME resolution in eyes with DME. ${ }^{24}$

Understanding why some eyes experience early, clinically meaningful DR improvements with anti-VEGF agents could provide important insights for both health care providers and patients as to the best time to initiate anti-VEGF therapy, and could help ensure the best outcomes for individual patients (personalized approach). To date, the few studies that have investigated the predictors of DR improvement with anti-VEGF therapy have focused on outcomes after 12 and 24 months of treatment. ${ }^{11,20,25}$

In this study, we investigated the clinical features and key baseline characteristics associated with early robust

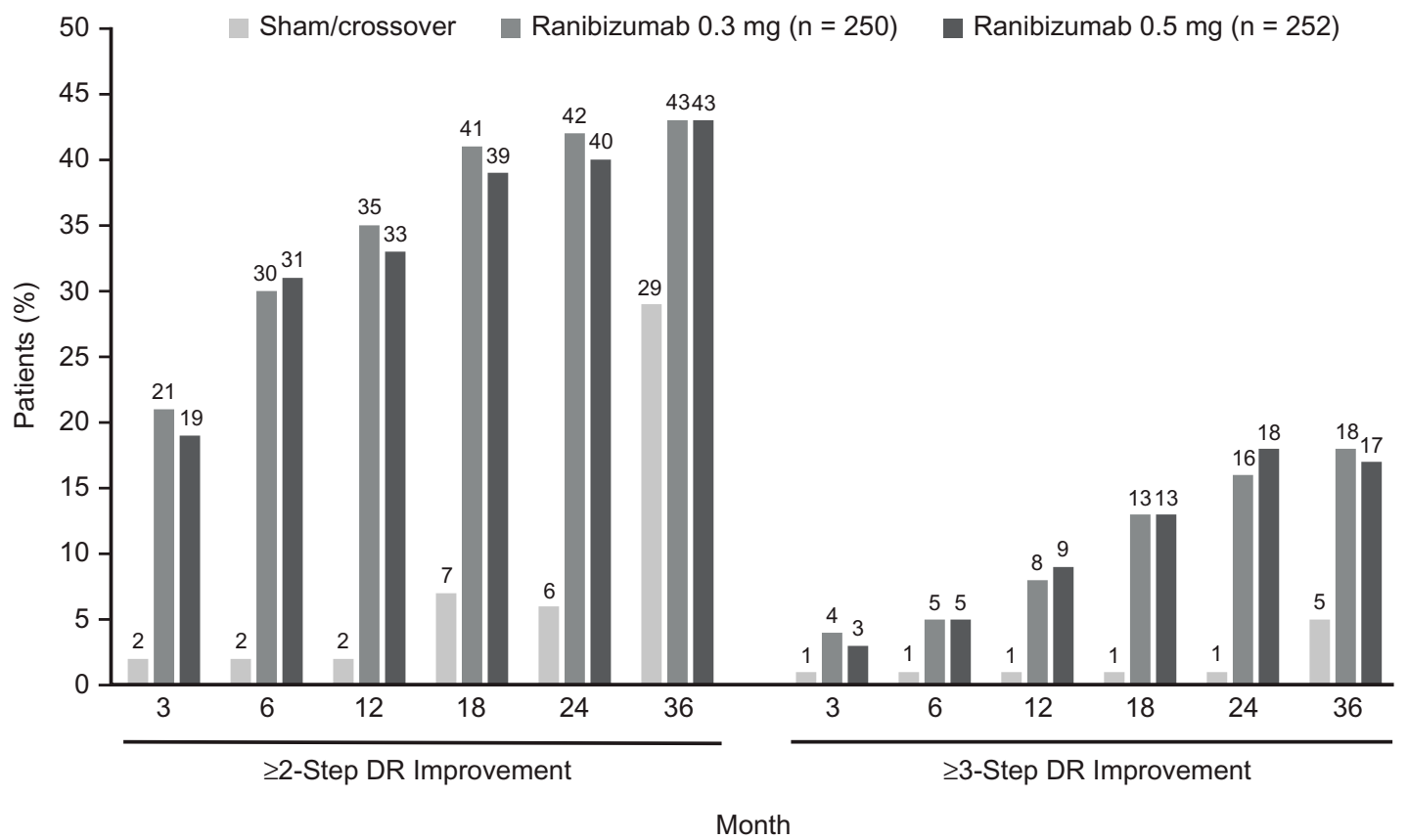

Figure I Bar graphs showing DR severity improvement with ranibizumab monthly therapy over the 36 months of the randomized phase of the RIDE/RISE trials (observed data pooled from both trials). The sham group received sham injections from baseline to month 24; patients then crossed over to ranibizumab 0.5 mg monthly from months 25-36. DR severity was assessed using the ETDRS-DRSS. Graph includes all patients, including those with no room for improvement due to low baseline ETDRS-DRSS scores. Reprinted from Ophthalmology, I26(5), Sun JK, Wang PW, Taylor S, Haskova Z. Durability of diabetic retinopathy improvement with as-needed ranibizumab: openlabel extension of RIDE and RISE studies, 7I2-720, Copyright (2019), with permission from Elsevier. ${ }^{21}$

Abbreviations: DR, diabetic retinopathy; DRSS, Diabetic Retinopathy Severity Scale; ETDRS, Early Treatment Diabetic Retinopathy Study. 
DR improvement during ranibizumab therapy in the RIDE/RISE clinical trials.

\section{Methods}

The study design and methods for the RIDE/RISE clinical trials (NCT00473382/NCT00473330) have been published and are described briefly below. ${ }^{23}$

RIDE/RISE were identical, Phase 3, prospective, multicenter, randomized, 36-month clinical trials. The studies adhered to the tenets of the Declaration of Helsinki and informed consent guidelines, and were in compliance with the regulations of the Health Insurance Portability and Accountability Act. The study protocols were prospectively approved by the institutional review board at each study site (Supplementary Table).

Adults with type 1 or 2 diabetes were eligible for enrollment if they had decreased vision due to DME in at least 1 eye. Patients across all levels of DR severity were included in the trials, ranging from DR severity 10 (no DR) through 75 (high-risk PDR). Approximately one-third of the patients had mild or moderate nonproliferative DR (NPDR), onethird had moderately severe or severe NPDR, and one-third had PDR, mostly mild and moderate. Less than 3.5\% of patients had high-risk PDR or questionable/no DR. Patients were excluded if they had unclear ocular media that would prevent capture of color fundus photographs or fluorescein angiograms. They were also excluded if they had any of the following interventions within 3 months of the screening visit: vitreoretinal surgery, panretinal photocoagulation (PRP) or macular laser, intraocular corticosteroids, or intravitreal antiangiogenic drugs. Patients with uncontrolled hypertension, uncontrolled diabetes, or recent (within 3 months) cerebrovascular accident or myocardial infarction were also excluded.

One eye per patient was designated as the study eye and randomized to monthly intravitreal ranibizumab $(0.5$ or $0.3 \mathrm{mg}$ ) or sham injections. All sham eyes crossed over to ranibizumab $0.5 \mathrm{mg}$ at month 24 . PRP was recommended in cases of high-risk PDR and permitted at the investigator's discretion in cases of severe NPDR or nonhigh-risk PDR. ${ }^{23}$

\section{Outcome Measures Relevant to the Present Analyses}

Eyes with evaluable color fundus photographs were assessed at baseline, month 3, and month 6 for DR severity by masked professional graders at an independent reading center (Fundus Photograph Reading Center at the University of Wisconsin, Madison, WI) using the ETDRSDRSS, a validated standardized scale originally developed and published by the ETDRS Research Group in 1991 and later adopted for use in assessments of DR severity changes in clinical trials. ${ }^{12}$ The scale includes the following grades: DRSS 10, 12 (DR absent), DRSS 14, 15, 20 (DR questionable), DRSS 35 (mild NPDR), DRSS 43 (moderate NPDR), DRSS 47 (moderately severe NPDR), DRSS 53 (severe NPDR), DRSS 61 (mild PDR), DRSS 65 (moderate PDR), DRSS 71 and 75 (high-risk PDR), DRSS 81 and 85 (advanced PDR). The scale is not linear and was originally designed to provide a tool to measure DR worsening and the associated increasing risk of vision loss. Therefore, the magnitude of change between individual steps on the scale qualitatively differ, even though they are assessed the same way when measured in a quantitative, numerical stepchange manner. The presence or absence of DME (defined as central subfield thickness $>250 \mu \mathrm{m}$ on time-domain optical coherence tomography) was also assessed at month 3 .

The proportion of eyes with an at least 2- or 3-step DR improvement on the ETDRS-DRSS from baseline to months 3 and 6 was assessed. Only eyes with room to improve were included in these analyses; a mild DRSS of at least 35 that improves by 2 steps (ie, to level 15) improves to 'DR questionable' status, while a moderate DRSS of at least 43 that improves by 2 steps (ie, to level 20 ) is still considered as having DR. Therefore, only eyes with a DRSS score of at least 43 underwent the 3-step evaluation. The number of eyes assessed for 2-step or greater DR improvement was 394 and 372 at months 3 and 6 , respectively. The number of eyes assessed for 3-step or greater DR improvement was 328 and 308 at months 3 and 6 , respectively.

\section{Data Analyses and Statistics}

Univariate analyses using Student's $t$-tests and chi-square tests were used to determine which baseline or early response characteristics were associated with 2-step or greater DR improvement in ranibizumab-treated patients. Characteristics evaluated included demographics, medical history, and systemic therapy, as well as ocular and systemic disease characteristics (Table 1). Note that a history of PRP was not included in the multivariable analysis of potential predictors because the presence of PRP scars automatically rendered a DR severity score of $\geq 60$ and prevented these eyes from being graded with a score $<60$, even if there was 
Table I Characteristics Assessed in Univariate Analyses for Association with Improvements in DR at Months 3 and 6

\begin{tabular}{|c|c|}
\hline Category & Characteristics \\
\hline Demographics & $\begin{array}{l}\text { - Age } \\
\text { - Sex } \\
\text { - Race } \\
\text { - Ethnicity }\end{array}$ \\
\hline Medical history & $\begin{array}{l}\text { - Diabetes duration } \\
\text { - HbAlc } \\
\text { - History of hypertension } \\
\text { - Hypertension duration } \\
\text { - History of hypercholesterolemia/hyperlipidemia } \\
\text { - Hypercholesterolemia/hyperlipidemia duration } \\
\text { - } \mathrm{BMI}\left(<30, \geq 30 \mathrm{~kg} / \mathrm{m}^{2}\right)\end{array}$ \\
\hline $\begin{array}{l}\text { Systemic } \\
\text { therapy }\end{array}$ & $\begin{array}{l}\text { - Diabetes medications (insulin alone, insulin plus } \\
\text { - } \text { other, other alone, no diabetes medication) } \\
\text { - Hypertension medications } \\
\text { - Thiazolidine medications }\end{array}$ \\
\hline $\begin{array}{l}\text { Ocular } \\
\text { characteristics }\end{array}$ & $\begin{array}{l}\text { - Baseline lens status } \\
\text { - DR severity at baseline }(35 / 43,47 / 53,60-75) \\
\text { - Months since DR or DME diagnosis } \\
\text { - Baseline BCVA ( } \leq 35,35-50,>50 \text { ETDRS letters) } \\
\text { - Baseline central foveal thickness on TD-OCT } \\
(\leq 400,>400 \mu \mathrm{m}) \\
\text { - Baseline macular nonperfusion } \\
\text { - DME status at month } 3\end{array}$ \\
\hline
\end{tabular}

Abbreviations: BCVA, best-corrected visual acuity; BMI, body mass index; DME, diabetic macular edema; DR, diabetic retinopathy; ETDRS, Early Treatment Diabetic Retinopathy Study; HbAlc, glycated hemoglobin; TD-OCT, time-domain optical coherence tomography.

an improvement in the retina surrounding the scars. Multivariable analyses were performed using logistic regression with backward selection, and included variables significant at the 0.05 significance level in the univariate analyses.

Analyses were completed with observed data for all variables. The ranibizumab 0.3 and $0.5 \mathrm{mg}$ treatment arms were pooled for analysis. Analyses were conducted using SAS software version 9.4 (SAS Institute Inc., Cary, NC).

\section{Results}

\section{Patient Population}

A total of 502 eyes were randomized to treatment with ranibizumab 0.3 or $0.5 \mathrm{mg}$ in the RIDE/RISE trials. ${ }^{23}$ Of these, 460 eyes had evaluable color fundus photographs at baseline.

Demographic characteristics at baseline for the eyes that were evaluated in the current analysis are shown in Table 2. The distribution of DR severities at baseline are shown in
Table 2 Baseline Characteristics of Analysis Cohort (Ranibizumab 0.3/0.5 mg-Treated Patients Only, Limited to DR Severity $\geq 35$ )

\begin{tabular}{|l|l|}
\hline & $\begin{array}{l}\text { Evaluable Eyes } \\
(\mathbf{N}=460)\end{array}$ \\
\hline $\begin{array}{l}\text { Age, years, mean (SD) } \\
\text { Sex, male, } \mathrm{n}(\%)\end{array}$ & $61.8(10.1)$ \\
$268(58.3)$
\end{tabular}

Note: *Due to missing data, the denominator is smaller than the analysis cohort for some variables.

Abbreviations: BCVA, best-corrected visual acuity; CST, central foveal thickness; $\mathrm{DR}$, diabetic retinopathy; HbAlc, glycated hemoglobin.

Table 3. DR severity scores at baseline ranged from 35 to 75 . A small proportion of patients with DR absent (0.5\%; DRSS $10,12)$ or questionable $(1.3 \%$; DRSS $14,15,20)$ were not included in the analyses due to lack of room for improvement. At baseline, $66.5 \%$ of patients had NPDR (DRSS $<60$ ) and $33.5 \%$ had PDR (DRSS $\geq 60$ ). Approximately $27 \%$ of the overall population had prior PRP.

The proportion of eyes in the pooled ranibizumab treatment groups with an at least 2- or 3-step improvement from baseline in ETDRS-DRSS at months 3 and 6 is shown in Figure 2. Note that a 2-step or greater DR improvement occurred in one-fifth of eyes at month 3 and in nearly one-third at month 6 .

The characteristics significantly associated $(P<0.05)$ with an at least 2- or 3-step DR improvement from baseline to months 3 and/or 6 in the univariate analyses are shown in Table 4. When factors significantly associated 
Table 3 DR Severity at Baseline

\begin{tabular}{|l|l|}
\hline $\begin{array}{l}\text { Baseline DR Severity (ETDRS-DRSS), } \\
\mathbf{n}(\%)\end{array}$ & $\begin{array}{l}\text { Evaluable Eyes } \\
\mathbf{( N = 4 6 0 )}\end{array}$ \\
\hline $\begin{array}{l}\text { Mild or moderate NPDR } \\
35\end{array}$ & $81(17.6)$ \\
43 & $63(13.7)$ \\
\hline Moderately severe or severe NPDR & \\
47 & $138(30.0)$ \\
53 & $24(5.2)$ \\
\hline PDR & \\
$60 / 6 \mathrm{I}$ & $133(28.9)$ \\
65 & $16(3.5)$ \\
71 & $4(0.9)$ \\
75 & $1(0.2)$ \\
\hline
\end{tabular}

Abbreviations: DR, diabetic retinopathy; DRSS, Diabetic Retinopathy Severity Scale; ETDRS, Early Treatment Diabetic Retinopathy Study; NPDR, nonproliferative diabetic retinopathy; PDR, proliferative diabetic retinopathy.

with a 2-step or greater DR improvement were evaluated using multivariable analysis, only baseline DR score was found to be predictive of a 2-step or greater improvement at both months 3 and 6 (Figure 3). At month 3, there were significantly $(P<0.0001)$ greater odds of 2 -step or greater improvement in eyes with moderately severe to severe NPDR compared with eyes with mild to moderate NPDR (odds ratio [OR], 24.4; 95\% confidence interval [CI], 7.4-83.3) or PDR (OR, 3.3; 95\% CI, 1.9-5.9; Figure 3A). Similarly, at month 6, a 2-step or greater improvement was significantly $(P<0.0001)$ more likely in eyes with moderately severe to severe NPDR than in eyes with mild to moderate NPDR (OR, 28.6; 95\% CI, 12.7-66.7) or PDR (OR, 15.4; 95\% CI, 8.0-29.4). The absence of DME at month 3 was also predictive of a 2-step or greater DR improvement at month $6(P=0.008)$; the OR (95\% CI) was $2.2(1.2-3.9)$ for DME absent versus DME present (Figure 3B).

Among eyes with a 2-step or greater DR improvement (79 eyes at month $3 ; 116$ eyes at month 6 ), the majority had moderately severe to severe NPDR at baseline (Figure 4A). Among eyes with a 3-step or greater DR improvement (12 eyes at month $3 ; 18$ eyes at month 6 ), the majority had PDR at baseline (Figure 4B).

Among eyes with a 2-step or greater DR improvement at month 6, 61.9\% had experienced resolution of DME at month 3 (Figure 5). A 2-step or greater DR improvement at month 6 was seen in $38.7 \%(70 / 181)$ of eyes that had resolution of DME at month 3 , but in only $23.5 \%(43 / 183)$ of eyes that had persistent DME at month 3. Among eyes with persistent DME at month 3, 70.9\% (139/196) still had DME at month 6.

\section{Discussion}

This study determined that the key predictive factor associated with early (month 3 and 6) robust DR regression in ranibizumab-treated eyes in the RIDE/RISE clinical trials was baseline DR severity score. The majority of eyes that experienced an early 2-step or greater DR improvement

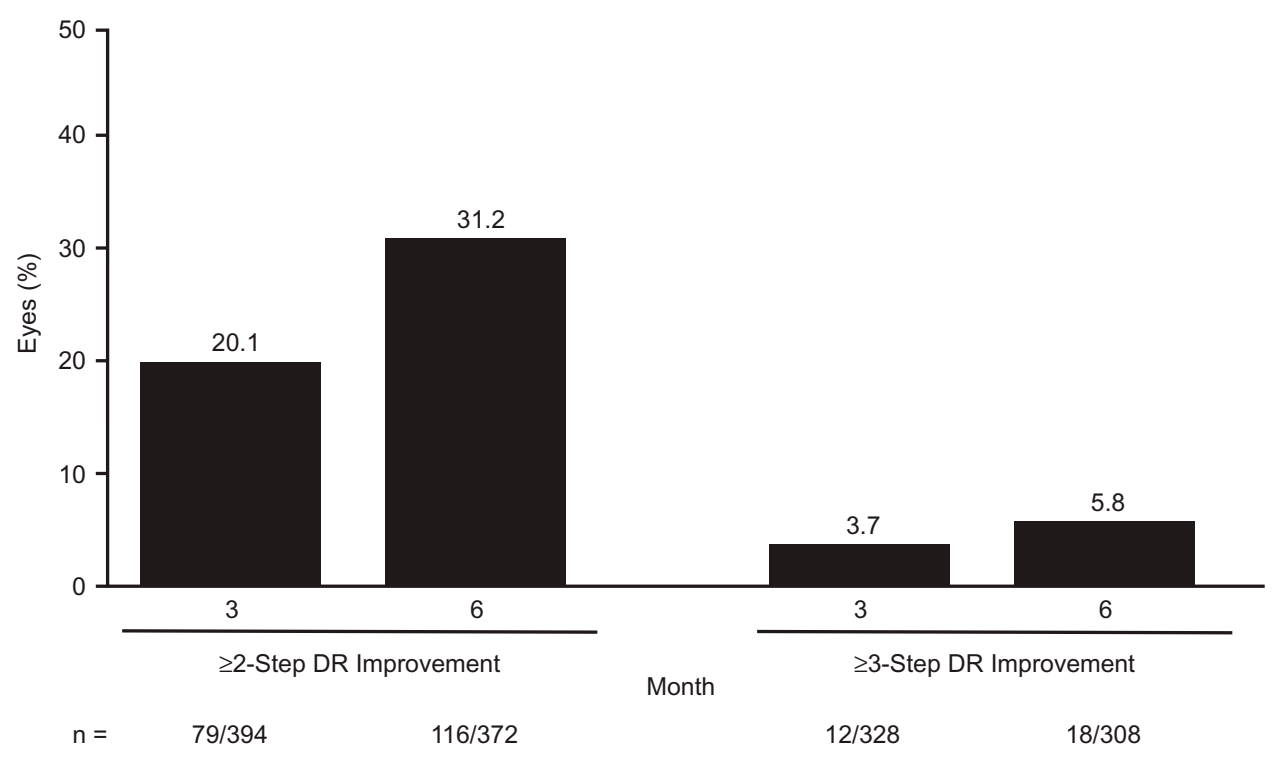

Figure 2 Bar graph showing early responders. Proportion of eyes treated with ranibizumab (0.3 and $0.5 \mathrm{mg}$ arms pooled) with an at least 2 - or 3 -step DR improvement from baseline at 3 and 6 months. DR severity was assessed using the ETDRS-DRSS. Only eyes with room to improve on the ETDRS-DRSS were included in this analysis. Abbreviations: DR, diabetic retinopathy; DRSS, Diabetic Retinopathy Severity Scale; ETDRS, Early Treatment Diabetic Retinopathy Study. 
Table 4 Characteristics Associated with Improvements in DR at Months 3 and 6 in the Univariate Analysis $(P<0.05)$

\begin{tabular}{|c|c|}
\hline Month 3 & Month 6 \\
\hline \multicolumn{2}{|l|}{ 2-Step or Greater Improvement } \\
\hline $\begin{array}{l}\text { - Age }(P=0.042) \\
\text { - } \text { Duration of diabetes }(P=0.046) \\
\text { - } \text { Duration of hypertension }(P=0.005) \\
\text { - } \text { Baseline DR severity }(P<0.000 \mathrm{I}) \\
\text { - } \text { Months since } D R \text { or } D M E \text { diagnosis } \\
(P=0.040) \\
\text { - Macular nonperfusion }(P=0.00 \mathrm{I})\end{array}$ & $\begin{array}{l}\text { - Age }(P=0.017) \\
\text { - } \text { Duration of diabetes }(P=0.003) \\
\text { - Baseline DR severity }(P<0.000 \mathrm{I}) \\
\text { - } \text { Months since } D R \text { or } D M E \text { diagnosis } \\
(P=0.00 \mathrm{I}) \\
\text { - DME at month } 3(P=0.002)\end{array}$ \\
\hline \multicolumn{2}{|l|}{ 3-Step or Greater Improvement } \\
\hline $\begin{array}{l}\text { - Black race }(P=0.003) \\
\text { - Baseline } D R \text { severity }(P=0.0 \mathrm{II})\end{array}$ & $\begin{array}{l}\text { - Age }(P=0.04 \mathrm{I}) \\
\text { - } \text { Baseline DR severity }(P=0.040) \\
\text { - } \text { Baseline BCVA }(P=0.010) \\
\text { - Macular nonperfusion }(P=0.038)\end{array}$ \\
\hline
\end{tabular}

Abbreviations: BCVA, best-corrected visual acuity; DME, diabetic macular edema; DR, diabetic retinopathy.

with ranibizumab at these timepoints had moderately severe to severe NPDR at baseline, and the majority of eyes with an early 3-step or greater DR improvement had PDR at baseline. Moreover, resolution of DME at month 3 was also predictive of a 2-step or greater DR improvement at month 6 , consistent with an earlier finding that improvements in DME and DR during ranibizumab treatment are correlated. $^{24}$

Our findings regarding the effect of baseline DR severity on the response to ranibizumab treatment are consistent with the results of other analyses of the RIDE/RISE trials that focused on later timepoints. Dhoot et al evaluated characteristics predictive of clinically meaningful DR improvements after 12 and 24 months of treatment with ranibizumab in RIDE/RISE and found that baseline DR severity was predictive of 2-step or greater DR improvement at both months 12 and $24 .^{25}$ Wykoff et al ${ }^{20}$ also evaluated the long-term outcomes from RIDE/RISE and found that the highest rate of 2-step or greater DR improvement at month 24 occurred in eyes with moderately severe to severe NPDR at baseline $78.4 \%$ in the ranibizumab $0.3 \mathrm{mg}$ group and $81.1 \%$ in the ranibizumab $0.5 \mathrm{mg}$ group). These improvements were seen as early as month 3 in approximately one-third of eyes with moderately severe to severe NPDR at baseline. ${ }^{20}$ Our new predictive analyses of early improvements, taken together with the past long-term results, suggest that it may be most beneficial to initiate anti-VEGF therapy in eyes with DR as soon as the condition has progressed to moderately severe or severe NPDR to ensure the greatest likelihood of both early and long-term robust improvements in DR. The present results also demonstrate, however, that eyes with PDR can still experience clinically meaningful improvements in DR severity with ranibizumab and should therefore also be considered for prompt treatment.

The present analysis also found that a 2-step or greater DR improvement at month 6 was more common among eyes with DME resolution by month 3 than in those who still had DME at month 3. Corresponding data, consistent with the results of our new predictive analysis, were reported by Ip et al, ${ }^{24}$ who found that resolution of DME and significant improvement in best-corrected visual acuity (BCVA) is more common in eyes with an at least 2- or 3-step DR improvement at month 24. In fact, additional analyses on RIDE/RISE data demonstrated that among eyes that had both resolution of DME and a 2-step or greater DR improvement at month 3, only $17.5 \%$ experienced an additional 2-step or greater DR improvement between months 3 and 24 (unpublished data). This is expected because most of these eyes had improved to the point that they no longer had room to improve by an additional 2 or more steps in DR severity. These data are in accord with and supportive of the RIDE/ RISE analyses published by Ip et al, ${ }^{24}$ which show that DR step improvements in patients with DME correlate with BCVA letter improvements, indicating the clinical importance of DR severity regression. Together, these findings show that there is a correlation between DME and DR responses to ranibizumab; this is consistent with the fact that both are associated with a VEGF-driven pathophysiology and that DME occurs as a complication of DR. Based on the existing data, we hypothesize that eyes that have early resolution of DME accompanied by early improvements in DR could be potentially good candidates for less frequent (less-than-monthly) ranibizumab treatment, while some eyes that do not have early resolution of DR and DME may benefit from continued treatment with antiVEGF therapy for up to 12-18 months (when the plateau of the population response is reached; Figure 1).

To the best of our knowledge, this is the first study to investigate the clinical features and key baseline characteristics associated with early robust DR regression with ranibizumab therapy. This analysis was made possible because the RIDE/RISE trials enrolled patients with a wide range of DR severities and collected high-quality color fundus photographs after as few as three or six 
A Month 3

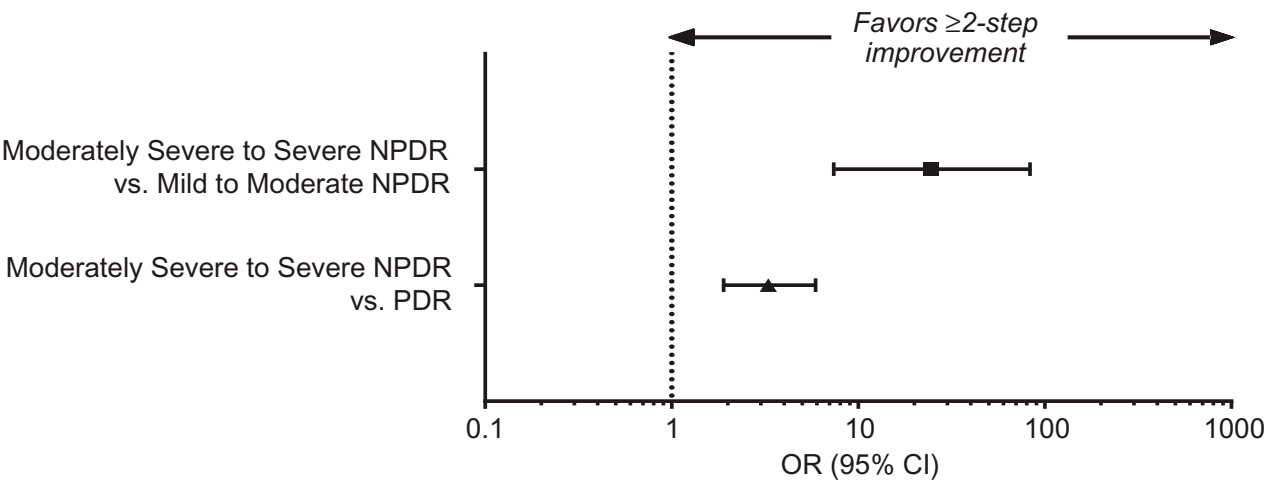

B Month 6

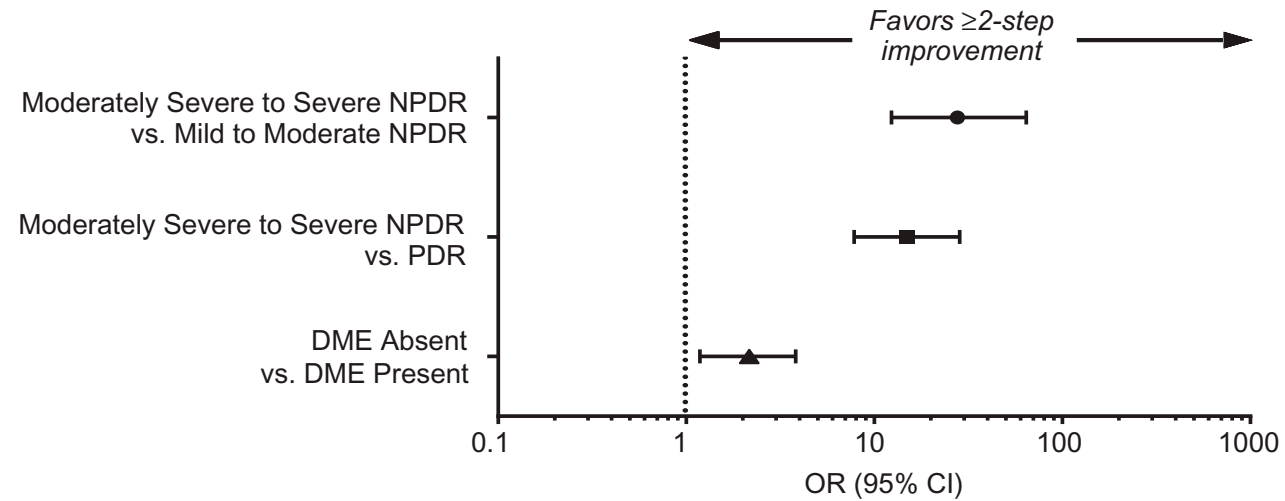

Figure 3 Forest plots showing factors predictive of early 2-step or greater DR improvement (multivariable analysis) at (A) month 3 and (B) month 6. Abbreviations: $\mathrm{Cl}$, confidence interval; DME, diabetic macular edema; DR, diabetic retinopathy; NPDR, nonproliferative diabetic retinopathy; OR, odds ratio; PDR, proliferative diabetic retinopathy.

monthly injections. Until recently, most studies of antiVEGF therapy in eyes with DR only collected fundus photographs at annual intervals. This was likely due to the perception of DR as a disease that would be unlikely to either progress or resolve during a shorter time period.

Another strength of this study was that DR outcomes were graded by two well-trained professional masked graders at a central reading center using the ETDRS-DRSS (a wellvalidated standardized method), with one senior grader serving as an arbitrator in cases of disagreement.

The key limitation of this study is that the patient population in the RIDE/RISE trials may not be directly representative of patients in the typical real-world clinical practice setting. The eligibility criteria required all enrolled patients to have decreased vision from DME in at least one eye, and excluded patients with conditions that could interfere with the collection or interpretation of the study results (eg, unclear ocular media, prior vitreoretinal surgery, recent history of PRP or macular laser). Patients were also required to have well-controlled diabetes (glycated hemoglobin $>12 \%),{ }^{23}$ which limited the ability to evaluate the relationship between glycated hemoglobin and early DR outcomes. Finally, the ETDRS-DRSS used for the evaluations of DR improvements in our analyses has limitations. The scale was not designed to assess DR improvements. It was originally designed to measure progressive DR worsening with associated increasing risk of vision loss. The scale is not linear and the individual DRSS steps differ in qualitative magnitude despite being assessed as the same quantitative difference. Inclusion of both qualitatively different disease states, NPDR and PDR, on the same linear scale could also be viewed as a limitation, especially when used for estimation of severity improvement in the absence of additional data, for example, extent of retinal ischemia. 
A Baseline DR Severity Distribution among Eyes with $\geq 2$-Step DR Improvement from Baseline

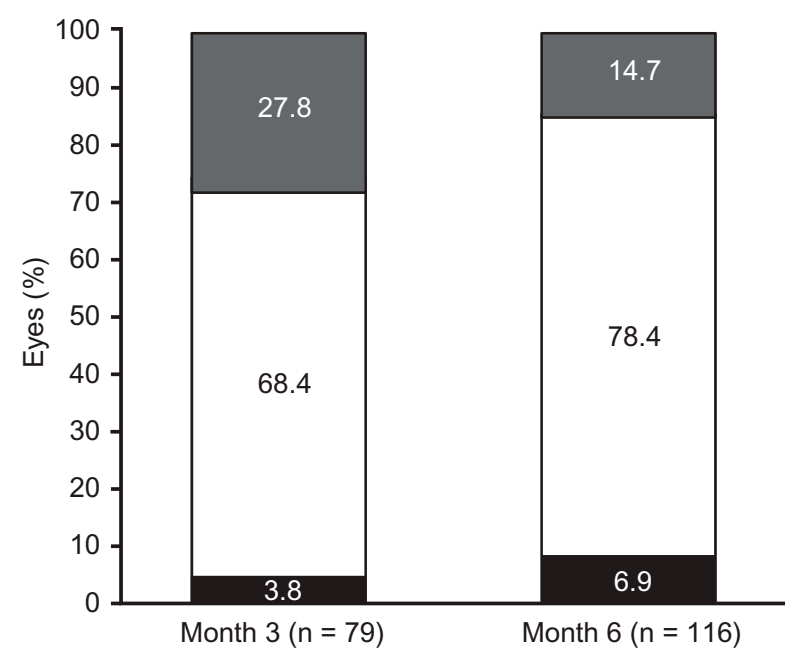

PDR (60-75) at baseline

$\square$ Moderately severe to severe NPDR $(47 / 53)$ at baseline

Mild to moderate NPDR (35/43) at baseline

B Baseline DR Severity Distribution among Eyes with $\geq 3$-Step DR Improvement from Baseline

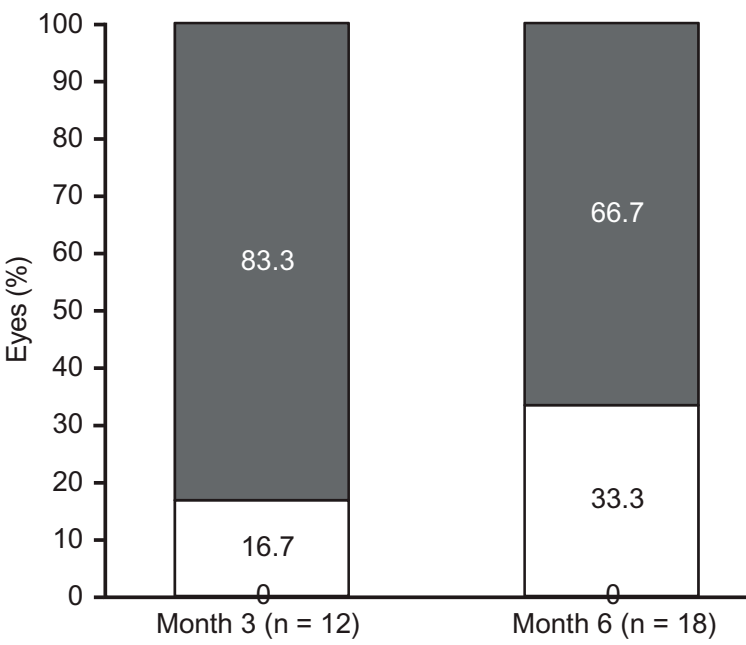

๑ PDR (60-75) at baseline

口 Moderately severe to severe NPDR $(47 / 53)$ at baseline

- Moderate NPDR (43) at baseline

Figure 4 Bar graphs showing the distribution of baseline DR severity among early responders with (A) 2-step or greater or (B) 3-step or greater DR improvement. DR severity assessed using the Early Treatment Diabetic Retinopathy Study Diabetic Retinopathy Severity Scale.

Abbreviations: DR, diabetic retinopathy; NPDR, nonproliferative diabetic retinopathy; PDR, proliferative diabetic retinopathy.

In conclusion, the present study demonstrates that patients with the highest risk of DR progression (moderately severe/ severe NPDR) and with the highest risk of vision-threatening complications (PDR) can achieve robust and rapid DR regression with ranibizumab treatment within the first few months of anti-VEGF treatment initiation. Interestingly, the majority of patients with the greatest magnitude of prompt 3-step or greater improvement at months 3 and 6 had PDR at baseline. These results highlight that it may not be too late for a pharmacological approach once eyes reach PDR, and eyes with PDR can have prompt and robust benefits with anti-
VEGF treatment. These rapid and clinically meaningful DR improvements with ranibizumab were more likely to occur in those eyes that also experienced early resolution of DME. The data highlight the importance of a personalized treatment approach in patients with diabetic eye disease, supporting previous findings that eyes that achieved the most benefit from anti-VEGF treatment were those with moderately severe to severe NPDR. ${ }^{20}$ Further investigations of optimal treatment frequency to maintain these robust and rapid improvements, including more durable treatments and sustained delivery options for treatment of DR, are warranted. 


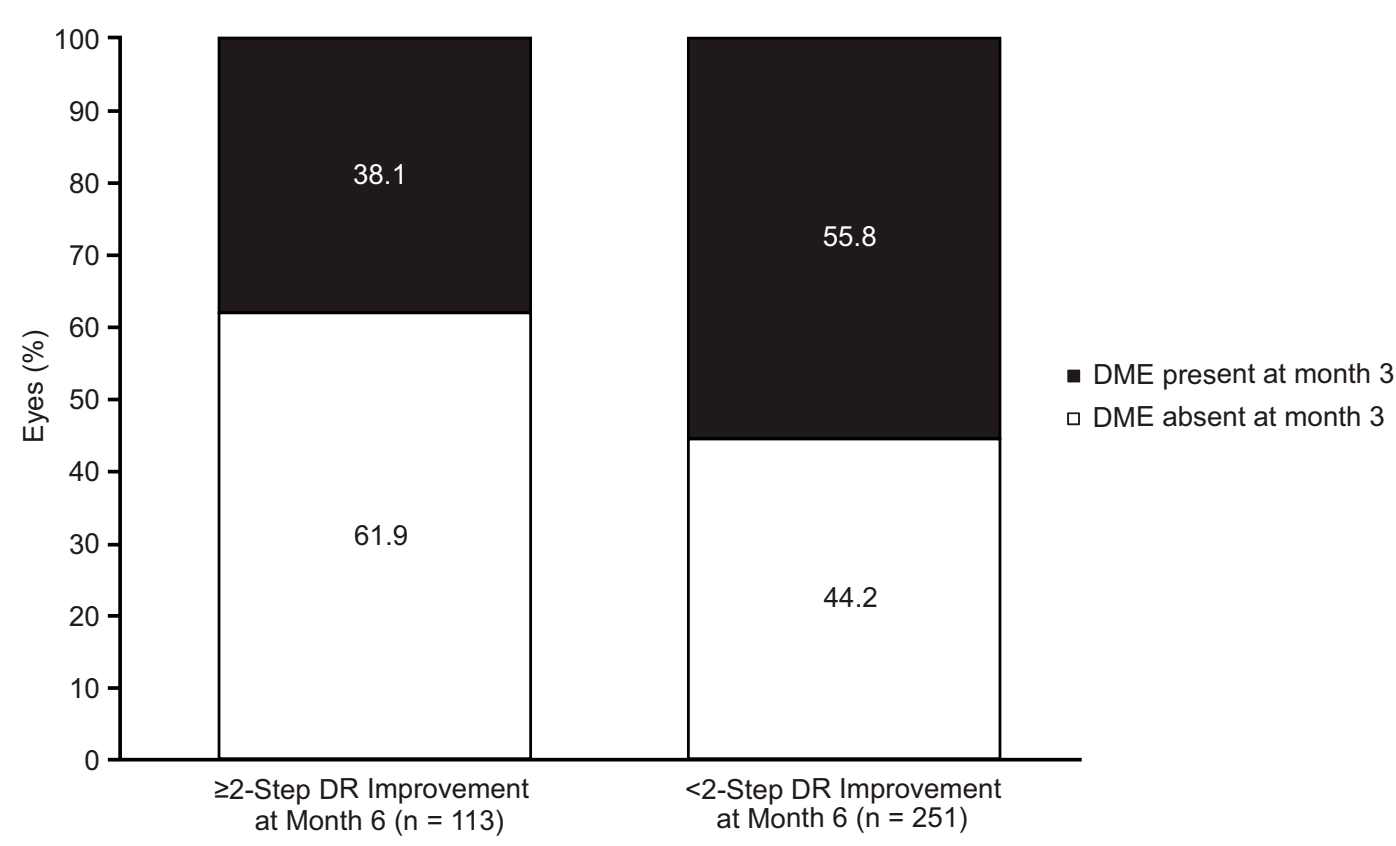

Figure 5 Bar graph showing the distribution of DME status at month 3 among eyes with or without a 2-step or greater improvement from baseline in DR at month 6 . DR severity assessed using the Early Treatment Diabetic Retinopathy Study Diabetic Retinopathy Severity Scale.

Abbreviations: DR, diabetic retinopathy; DME, diabetic macular edema.

\section{Abbreviations}

BCVA, best-corrected visual acuity; CI, confidence interval; DME, diabetic macular edema; DR, diabetic retinopathy; DRSS, Diabetic Retinopathy Severity Scale; ETDRS, Early Treatment Diabetic Retinopathy Study; NPDR, nonproliferative diabetic retinopathy; OR, odds ratio; PDR, proliferative diabetic retinopathy; PRP, panretinal photocoagulation; VEGF, vascular endothelial growth factor.

\section{Data Sharing Statement}

Qualified researchers may request access to individual patient level data through the clinical study data request platform (https://vivli.org/). Further details on Roche's criteria for eligible studies are available here (https://vivli. org/members/ourmembers/). For further details on Roche's Global Policy on the Sharing of Clinical Information and how to request access to related clinical study documents, see here (https://www.roche.com/research_and_develop ment/who we are how we work/clinical trials/our_com mitment_to_data_sharing.htm).

\section{Acknowledgments}

Third-party writing assistance, which was provided by Amy Lindsay, $\mathrm{PhD}$, of Envision Pharma Group, was funded by Genentech, Inc. Portions of these data were presented at the 41st Annual Macula Society Meeting, February 21-24, 2018, Beverly Hills, CA, USA; the American Society of Retina Specialists Annual Meeting, July 20-25, 2018, Vancouver, BC, Canada; the 18th Congress of the European Society of Retina Specialists, September 20-23, 2018, Vienna, Austria; and the Annual American Academy of Optometry Meeting, November 7-10, 2018, San Antonio, TX, USA.

\section{Author Contributions}

All authors contributed to the interpretation of the data in this manuscript, provided critical revisions to the manuscript for important intellectual content, approved the final version of the manuscript to be published, and agree to be accountable for all aspects of the work in ensuring that questions related to the accuracy or integrity of any part of the work are appropriately investigated and resolved. In addition, MS, MH, LH, LT, and ZH contributed to study conception and design; and LH conducted the statistical analyses.

\section{Funding}

Genentech, Inc., South San Francisco, CA, USA, provided support for the study and participated in the study design; conducting the study; and data collection, management, and interpretation. 


\section{Disclosure}

MS is a consultant and/or speaker for Aerpio, Allegro, Allergan, Alimera, Appellis, Clearside, Eyepoint, Genentech, Inc., Guidepoint, Ionis, Kodiak, Mallinckrodt, Notal Vision, Novartis, Opthea, Optos, Regeneron, Roche, Santen, and Spark. ML is an advisor for Genentech, Inc. and reports personal fees from Roche, outside the submitted work. PS is an advisor for Bayer, Novartis, and Roche; and has received grants from Allergan, Bayer, and Novartis. AMK is an investigator for Adverum, Aerpio, Allergan, Apellis, Clearside, Genentech, Inc., Novartis, Opthea, Oxurion, Ophthotech, Recens, and Regenxbio; and a consultant and/or speaker for Aerpio, Allergan, Genentech, Inc., Kodiak, Notal Vision, Novartis, Opthea, Oxurion, PolyPhotonix, and Roche. LH is a contractor for Genentech, Inc. MH, LT, and ZH are full-time employees of Genentech, Inc. ZH received professional medical writing support from Envision Pharma Group. The authors report no other conflicts of interest in this work.

\section{References}

1. Centers for Disease Control and Prevention. Common eye disorders. Available from: https://www.cdc.gov/visionhealth/basics/ced/index. html. Accessed November 3, 2018.

2. Ciulla TA, Amador AG, Zinman B. Diabetic retinopathy and diabetic macular edema: pathophysiology, screening, and novel therapies. Diabetes Care. 2003;26(9):2653-2664. doi:10.2337/diacare.26.9.2653

3. Gupta N, Mansoor S, Sharma A, et al. Diabetic retinopathy and VEGF. Open Ophthalmol J. 2013;7:4-10. doi:10.2174/1874364101307010004

4. Nathan DM, Genuth S, Lachin J, et al; Diabetes Control and Complications Trial Research Group. The effect of intensive treatment of diabetes on the development and progression of long-term complications in insulin-dependent diabetes mellitus. $N$ Engl $J$ Med. 1993;329(14):977-986. doi:10.1056/NEJM199309303291401

5. Diabetes Control and Complications Trial Research Group. Progression of retinopathy with intensive versus conventional treatment in the Diabetes Control and Complications Trial. Ophthalmology. 1995;102 (4):647-661. doi:10.1016/s0161-6420(95)30973-6

6. UK Prospective Diabetes Study (UKPDS) Group. Intensive blood-glucose control with sulphonylureas or insulin compared with conventional treatment and risk of complications in patients with type 2 diabetes (UKPDS 33). Lancet. 1998;352(9131):837-853. doi:10.1016/S0140-6736(98)07019-6

7. Aiello LP, Avery RL, Arrigg PG, et al. Vascular endothelial growth factor in ocular fluid of patients with diabetic retinopathy and other retinal disorders. $N$ Engl $J$ Med. 1994;331(22):1480-1487. doi:10.1056/NEJM199412013312203

8. Funatsu H, Yamashita H, Sakata K, et al. Vitreous levels of vascular endothelial growth factor and intercellular adhesion molecule 1 are related to diabetic macular edema. Ophthalmology. 2005;112 (5):806-816. doi:10.1016/j.ophtha.2004.11.045

9. Mahdy RA, Nada WM, Hadhoud KM, El-Tarhony SA. The role of vascular endothelial growth factor in the progression of diabetic vascular complications. Eye (Lond). 2010;24(10):1576-1584. doi:10.1038/ eye. 2010.86
10. Brown DM, Schmidt-Erfurth U, Do DV, et al. Intravitreal aflibercept for diabetic macular edema: 100-week results from the VISTA and VIVID studies. Ophthalmology. 2015;122(10):2044-2052. doi:10.1016/j.ophtha. 2015.06.017

11. Dhoot DS, Baker K, Saroj N, et al. Baseline factors affecting changes in Diabetic Retinopathy Severity Scale score after intravitreal aflibercept or laser for diabetic macular edema: post hoc analyses from VISTA and VIVID. Ophthalmology. 2018;125(1):51-56. doi:10.1016/j.ophtha.2017. 06.029

12. Ip MS, Domalpally A, Hopkins JJ, Wong P, Ehrlich JS. Long-term effects of ranibizumab on diabetic retinopathy severity and progression. Arch Ophthalmol. 2012;130(9):1145-1152. doi:10.10 01/archophthalmol.2012.1043

13. Adamis AP, Altaweel M, Bressler NM, et al; Macugen Diabetic Retinopathy Study Group. Changes in retinal neovascularization after pegaptanib (Macugen) therapy in diabetic individuals. Ophthalmology. 2006;113(1):23-28. doi:10.1016/j.ophtha.2005.10.012

14. Brown DM, Nguyen QD, Marcus DM, et al; RIDE and RISE Research Group. Long-term outcomes of ranibizumab therapy for diabetic macular edema: the 36-month results from two phase III trials: RISE and RIDE. Ophthalmology. 2013;120(10):2013-2022. doi:10.1016/j.ophtha.2013.02.034

15. Bressler SB, Qin H, Melia M, et al; Diabetic Retinopathy Clinical Research Network. Exploratory analysis of the effect of intravitreal ranibizumab or triamcinolone on worsening of diabetic retinopathy in a randomized clinical trial. JAMA Ophthalmol. 2013;131 (8):1033-1040. doi:10.1001/jamaophthalmol.2013.4154

16. Schmidt-Erfurth U, Lang GE, Holz FG, et al; RESTORE Extension Study Group. Three-year outcomes of individualized ranibizumab treatment in patients with diabetic macular edema: the RESTORE extension study. Ophthalmology. 2014;121(5):1045-1053. doi:10.1016/j. ophtha.2013.11.041

17. Boyer DS, Nguyen QD, Brown DM, Basu K, Ehrlich JS; RIDE and RISE Research Group. Outcomes with as-needed ranibizumab after initial monthly therapy: long-term outcomes of the phase III RIDE and RISE trials. Ophthalmology. 2015;122(12):2504-2513.e1. doi:10.1016/j.ophtha.2015.08.006

18. Bressler SB, Liu D, Glassman AR, et al; Diabetic Retinopathy Clinical Research Network. Change in diabetic retinopathy through 2 years: secondary analysis of a randomized clinical trial comparing aflibercept, bevacizumab, and ranibizumab. JAMA Ophthalmol. 2017;135(6):558-568. doi:10.1001/jamaop hthalmol.2017.0821

19. Gross JG, Glassman AR, Jampol LM, et al; Writing Committee for the Diabetic Retinopathy Clinical Research Network. Panretinal photocoagulation vs intravitreous ranibizumab for proliferative diabetic retinopathy: a randomized clinical trial. JAMA. 2015;314 (20):2137-2146. doi:10.1001/jama.2015.15217

20. Wykoff CC, Eichenbaum DA, Roth DB, Hill L, Fung AE, Haskova Z. Ranibizumab induces regression of diabetic retinopathy in most patients at high risk of progression to proliferative diabetic retinopathy. Ophthalmol Retina. 2018;2(10):997-1009. doi:10.1016/j. oret.2018.06.005

21. Sun JK, Wang PW, Taylor S, Haskova Z. Durability of diabetic retinopathy improvement with as-needed ranibizumab: open-label extension of RIDE and RISE studies. Ophthalmology. 2019;126 (5):712-720. doi:10.1016/j.ophtha.2018.10.041

22. Wykoff CC, Ou WC, Khurana RN, Brown DM, Lloyd Clark W, Boyer DS; ENDURANCE Study Group. Long-term outcomes with as-needed aflibercept in diabetic macular oedema: 2-year outcomes of the ENDURANCE extension study. Br J Ophthalmol. 2018;102 (5):631-636. doi:10.1136/bjophthalmol-2017-310941 
23. Nguyen QD, Brown DM, Marcus DM, et al; RISE and RIDE Research Group. Ranibizumab for diabetic macular edema: results from 2 phase III randomized trials: RISE and RIDE. Ophthalmology. 2012;119(4):789-801. doi:10.1016/j.ophtha.2011.12.039

24. Ip MS, Zhang J, Ehrlich JS. The clinical importance of changes in Diabetic Retinopathy Severity Score. Ophthalmology. 2017;124 (5):596-603. doi:10.1016/j.ophtha.2017.01.003
25. Dhoot D, Hill L, Tarnowski K, Stoilov I. Baseline factors associated with $\geq 2$-step diabetic retinopathy (DR) severity improvement with ranibizumab (RBZ). Invest Ophthalmol Vis Sci. 2018;59(9):3592. doi: $10.1167 /$ iovs. 17-23678

\section{Publish your work in this journal}

Clinical Ophthalmology is an international, peer-reviewed journal covering all subspecialties within ophthalmology. Key topics include: Optometry; Visual science; Pharmacology and drug therapy in eye diseases; Basic Sciences; Primary and Secondary eye care; Patient Safety and Quality of Care Improvements. This journal is indexed on PubMed

Submit your manuscript here: https://www.dovepress.com/clinical-ophthalmology-journal
Central and CAS, and is the official journal of The Society of Clinical Ophthalmology (SCO). The manuscript management system is completely online and includes a very quick and fair peer-review system, which is all easy to use. Visit http://www.dovepress.com/ testimonials.php to read real quotes from published authors. 